\title{
Estimation of COVID-19-induced depletion of hospital resources in Ontario, Canada
}

\author{
Kali Barrett MD, Yasin A. Khan MD, Stephen Mac MBiotech, Raphael Ximenes PhD, David M.J. Naimark MD MSc, \\ Beate Sander PhD
}

Cite as: CMAJ 2020 June 15;192:E640-6. doi: 10.1503/cmaj.200715; early-released May 14, 2020

See related articles at www.cmaj.ca/lookup/doi/10.1503/cmaj.200457 and at www.cmaj.ca/lookup/doi/10.1503/cmaj.200476

\begin{abstract}
BACKGROUND: The global spread of coronavirus disease 2019 (COVID-19) continues in several jurisdictions, causing substantial strain to health care systems. The purpose of our study was to predict the effect of the COVID-19 pandemic on patient outcomes and use of hospital resources in Ontario, Canada.
\end{abstract}

METHODS: We developed an individuallevel simulation to model the flow of patients with COVID-19 through the hospital system in Ontario. We simulated different combined scenarios of epidemic trajectory and hospital health care capacity. Our outcomes included the number of patients who needed admission to the ward or to the intensive care unit (ICU) with or without the need for mechanical ventilation, number of days to resource depletion, number of patients awaiting resources and number of deaths.

RESULTS: We found that with effective early public health measures, hospital system resources would not be depleted. For scenarios with late or ineffective implementation of physical distancing, hospital resources would be depleted within 14-26 days, and in the worst case scenario, 13321 patients would die while waiting for needed resources. Resource depletion would be avoided or delayed with aggressive measures to increase ICU, ventilator and acute care hospital capacities.

INTERPRETATION: We found that without aggressive physical distancing measures, the Ontario hospital system would have been inadequately equipped to manage the expected number of patients with COVID-19 despite a rapid increase in capacity. This lack of hospital resources would have led to an increase in mortality. By slowing the spread of the disease using public health measures and by increasing hospital capacity, Ontario may have avoided catastrophic stresses to its hospitals.

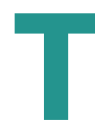

he global spread of severe acute respiratory syndrome coronavirus 2 (SARS-CoV-2) and the resulting clinical syndrome, coronavirus disease 2019 (COVID-19), was declared a pandemic on Mar. 11, 2020.1,2 As of Apr. 26, 2020, 2.9 million confirmed cases and close to 200000 deaths have been reported worldwide. ${ }^{3,4}$ Alarmingly, the first 100000 cases developed over 3 months, and the subsequent 100000 cases developed over only 12 days. ${ }^{5}$ To decrease the spread of COVID19 , much of the global community mobilized efforts to minimize social interactions (i.e., physical distancing), and some countries pursued aggressive testing and contact tracing. ${ }^{6}$ Without measures to decrease the rate of spread, patients' needs for critical care will overwhelm available resources. ${ }^{7,8}$

Predicting COVID-19 population spread and assessing interventions aiming to mitigate transmission are important to ensure that health care systems are adequately prepared for the ongoing pandemic. Several transmission models suggested that without aggressive physical distancing, the need for hospital admission and critical care would outstrip available resources. ${ }^{9,10}$ Although such transmission models help to describe the size of COVID-19 spread under different scenarios and provide high-level estimates of resource use, they do not incorporate resource constraints dynamically (i.e., cases approach the resource threshold in a linear fashion). These models are unable to account for changes in resource constraints owing to the consequences of death and recovery, queuing and setting priorities among patients waiting in queue.

With COVID-19, evidence suggests that lower hospital capacity is associated with higher mortality. ${ }^{11}$ Understanding how COVID19 will affect hospital resources is especially relevant in Canada: in 2018 , there were 2.5 hospital beds per 1000 population compared with 4.34, 3.84, 3.18 and 2.54 beds per 1000 population in China, Australia, Italy and the United Kingdom, respectively. ${ }^{12}$

Our objective was to predict the effect of COVID-19 on the hospital system and mortality in Ontario, Canada, for a range of COVID-19 cases and levels of hospital capacity, to show what might have happened to Ontario's hospital system in worst-case and best-case scenarios, and what we expect the actual effect in Ontario to be. 


\section{Methods}

\section{Study design and data sources}

We developed a discrete-time, individual-level, health-state transition model to forecast hospital resource use for patients with COVID-19 presenting to hospital. Primary outcomes were number of patients needing admission to the ward or intensive care unit (ICU) - with or without the need for mechanical ventilation; days to depletion of any of the resources; number of patients waiting for any of the resources per day; and number of COVID-19-related deaths among patients admitted to hospital, stratified by whether or not patients received the needed resource. Outcomes were accrued over a time horizon of 60 days $(2 \mathrm{mo})$, and the cycle length (time steps) of the model was denominated in days.

We simulated a dynamic population of adults (18 $\mathrm{yr}$ and older) with symptomatic COVID-19 infection who arrived at the hospital emergency department, where they were either sent home to self-isolate or admitted (Figure 1). Hospitalized patients were admitted to a general medical ward or directly to the ICU depending on disease severity, and some ICU patients required invasive mechanical ventilation. Throughout the model, patients could either remain in their health state, recover or die.

If any of the resources (i.e., ward beds, ICU beds and ventilators) were unavailable, we assumed that the patient remained in their current place (resource state) waiting for the resource to become available. For example, if a patient needed an ICU bed and none were available, the patient would remain in the emergency department with no access to critical care resources until an ICU bed was available. Ventilators and ICU beds were freed up upon recovery or death of patients. Ward beds were freed up upon recovery of patients. We determined priority setting for ward beds by the patient's current location (ICU patients were prioritized over patients in the emergency department). For other resources, priority was given to patients who were waiting the longest since admission. We conducted all modelling and analyses using TreeAge Pro 2020 (TreeAge Software).

\section{COVID-19 disease trajectory}

Data were extracted from the evolving literature on COVID-19, supplemented by expert guidance (Table 1 ).

We assumed that the probability of admission to hospital in Ontario was similar to crude Canadian estimates for hospital admission of $18 \%$ for symptomatic adults with SARS-CoV- 2 infection. ${ }^{13}$ In the absence of Ontario-specific data, we estimated ICU admission given admission to hospital to be $48 \%$ by grid calibration to observed ICU occupancy. We used a rudimentary grid search because more systematic calibration techniques, such as simulated annealing or the Nelder-Mead algorithm, presuppose the existence of high-quality observed data, which, at this point, were not available. The Ontario estimate for ICU admissions requiring ventilation was $78 \%$, as reported by Critical Care Services Ontario on Apr. 13, $2020 .{ }^{14}$ We assumed that only patients requiring ICU admission had a risk of death during their hospital stay. ${ }^{11}$ We assumed the same mortality risk for patients who required intensive care without ventilation but could not access ICU beds owing to resource constraints. We assumed that patients who needed mechanical ventilation but

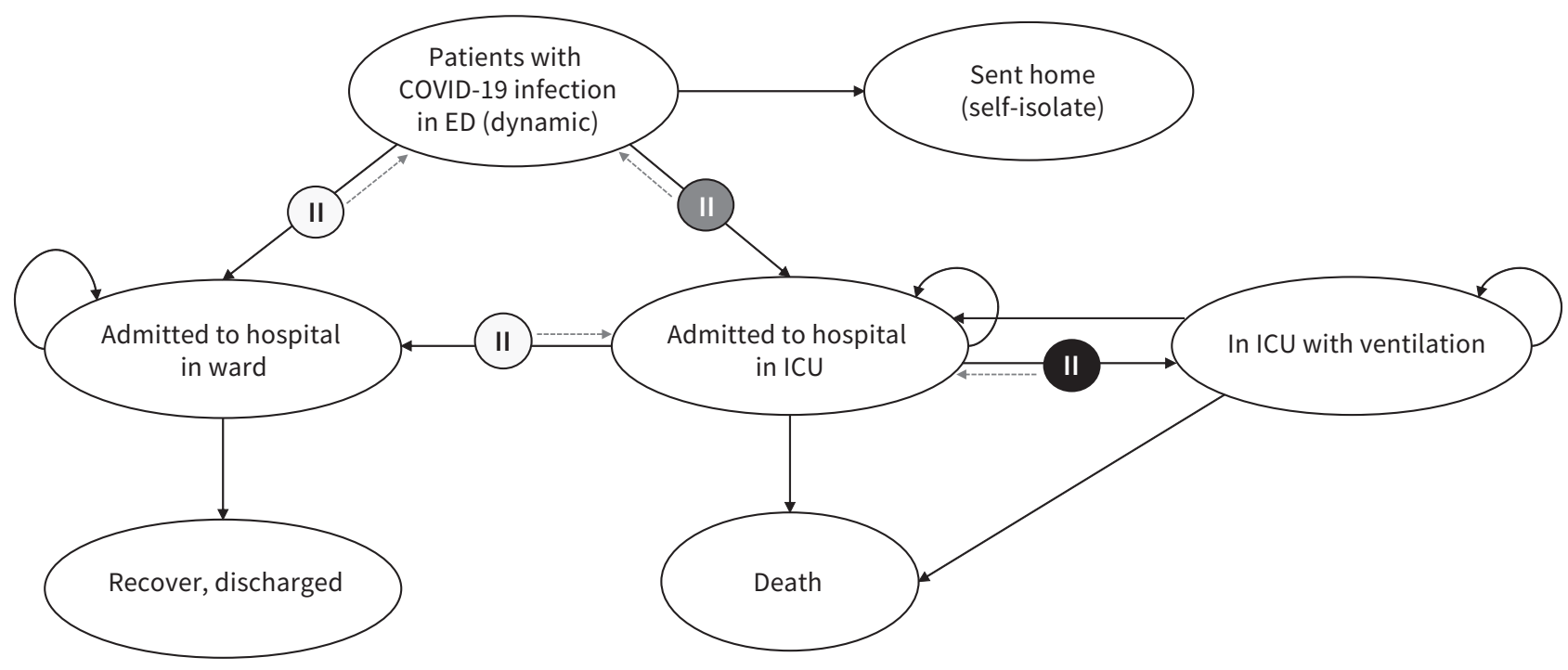

(II) This stop node represents resource constraint for hospital beds. If there are no more resources, the patient will remain in that previous state as indicated by the dashed line.

II This stop node represents resource constraint for ICU beds. If there are no more resources, the patient can remain in that previous state as indicated by the dashed line.

II This stop node represents resource constraint for ventilators. If there are no more resources, the patient will remain in that previous state as indicated by the dashed line, and was assumed to die within the day.

Figure 1: Model schematic. COVID-19 = coronavirus disease 2019, ED = emergency department, ICU = intensive care unit. 
could not access it would die that day. The probability of death in the ICU (for patients requiring and not requiring mechanical ventilation) was based on the proportion of deaths (35\%) for patients with moderate acute respiratory distress syndrome (ARDS), who had a mean length of ICU stay of 11 days (and a mean hospital length of stay of $17 \mathrm{~d}$ ), which was reported in a study involving several countries. ${ }^{15}$ We used length of stay and mortality estimates from patients with moderate ARDS because they are considered clinically similar to COVID-19 cases in the ICU, according to expert guidance.

\section{Exploring COVID-19's effect on availability of hospital} resources

In the absence of effective specific treatments for COVID-19, desirable health outcomes can be maximized by reducing the number of cases through public health measures and by increasing system capacity (i.e., freeing up existing resources or adding additional resources). We explored 9 scenarios that considered COVID-19 spread and system capacity: 3 possible epidemic trajectories and 3 resource availability scenarios.

\section{Table 1: Key variables for modelling}

Variable

Number of patients with COVID-19 reported in Ontario, per day, by scenario

Probability of needing admission to hospital

Probability of needing ICU-level care given admission to hospital

Probability of patients in ICU needing ventilation

Probability that patients on the ward deteriorate and need

ICU-level care

Length of stay, ward (no ICU admission)

Length of stay, ICU (with or without ventilation)

Length of stay, ward after a stay in ICU

Probability of death, ward patients

Probability of death, ICU patients

Probability of death, patients with ventilation

Probability of death, patients waiting for ventilation
Base-case

value

Source

See Figure 2 Estimated as described in text

$\begin{array}{cl}0.18 & \text { Public Health Agency of Canada estimate } \\ 0.48 & \text { Calibrated based on Public Health Agency of Canada estimate }{ }^{13} \\ 0.78 & \text { CCSO estimate on Apr. 13, 2020 } \\ 0 & \text { Assumption } \\ 17 \text { d } & \text { Bellani et al., } 2016^{15} \\ 11 \mathrm{~d} & \text { Bellani et al., } 2016^{15} \\ 6 \mathrm{~d} & \text { Bellani et al., } 2016^{15} \\ 0 & \text { Assumption guided by Wu and McGoogan 2020 } \\ 0.35 & \text { Bellani et al., } 2016^{15} \\ 0.35 & \text { Bellani et al., } 2016^{15} \\ 1.0 & \text { Assumption }\end{array}$

Note: $\mathrm{CCSO}=$ Critical Care Services Ontario, COVID-19 = coronavirus disease 2019, ICU = intensive care unit.

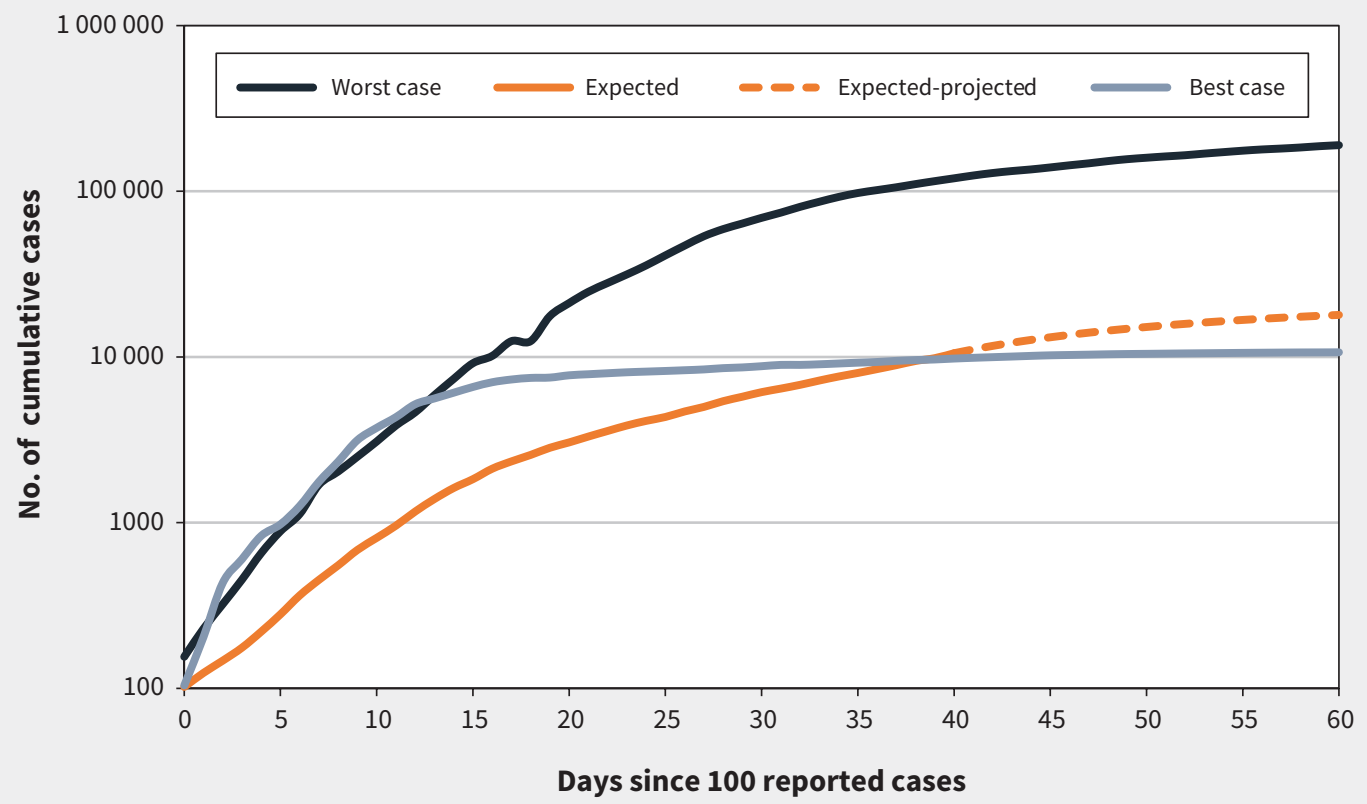

Figure 2: Cumulative number of new coronavirus disease 2019 (COVID-19) cases over time in Ontario. 


\section{Epidemic trajectory scenarios}

We modelled 3 scenarios of possible epidemic trajectory in Ontario starting on Mar. 5, 2020, when Ontario had reported 102 cumulative cases of COVID-19, defined as confirmed reported cases (i.e., a positive test result for SARS-CoV-2).

\section{Best-case scenario}

We used observed daily incidence data from South Korea for 60 days (reported data to Apr. 23, 2020), ${ }^{3}$ starting at 104 cumulative cases, which was aligned to Ontario's starting date.

\section{Expected scenario}

Starting at 102 cumulative cases on Mar. 5, 2020, we used observed data from Ontario's integrated Public Health Information System until Apr. 14 to account for reporting delays, then applied a 5\% daily decrease (contraction rate) in new cases per day until day 60 . A peak was observed on Apr. 13; the contraction rate represents the effect of public health measures enacted in mid-March.

\section{Worst-case scenario}

We used observed daily incidence data from Italy for 60 days (reported data to Apr. 23, 2020), ${ }^{3}$ starting at 155 cumulative cases, which was best aligned to Ontario's starting date.

Case trajectories are shown in Figure 2. Daily numbers of expected cases can be found in Appendix 1 (available at www. cmaj.ca/lookup/suppl/doi:10.1503/cmaj.200715/-/DC1).

\section{Hospital resource scenarios}

We considered ward beds and ICU beds with or without a ventilator. For a patient to receive mechanical ventilation, both a ventilator and ICU bed needed to be available. We assumed all available resources were appropriately staffed. We used 3 resource scenarios in our modelling: base-case, surge capacity and vast increase.

\section{Base-case scenario}

The number of available ventilator beds at the start of the simulation represents the number of existing ventilators in Ontario. We assumed that $25 \%$ of total existing ventilator beds (328/1311) and ICU beds without ventilators (186/742), and $20 \%$ of hospital ward beds (4000/20000) were available for patients with COVID-19 based on expert judgment. We assumed that no additional beds or ventilators would be made available for COVID-19 patients.

\section{Surge scenario}

We added a surge capacity of 502 beds with ventilators, 351 ICU beds without ventilators and 1351 ward beds to the base case (total no.: 830 beds with ventilators, 537 ICU beds without ventilators and 5351 ward beds). This scenario maximized currently available resources through a reduction in clinical activity and activation of existing surge capacity protocols (Erik Hellsten, Ontario Health, Toronto: personal communication, 2020).

\section{Vast-increase scenario}

In this scenario, there was a vast increase in health system capacity through unconventional hospital space. Therefore, 2000 ICU beds with ventilators, 1000 ICU beds without ventilators and 10000 ward beds would be available to patients with COVID-19.

\section{Results}

\section{Epidemic trajectory scenarios}

Our best-case epidemic trajectory scenario, assuming base-case resources, predicted a total of 10579 COVID-19 cases over 60 days, of which 1904 required admission to hospital (Table 2). Although ICU beds, ventilator resources and ward beds in Ontario would be used extensively between days 10 and 20 because of the rapid increase in COVID-19 cases, they would never be depleted

\section{Table 2: Relation between epidemic trajectory, resource availability and mortality}

No. of days until resource was unavailable

Resource
scenario

Base case

Best case

Expected

Worst case

ICU beds

Ventilators

Never

Never

Never

Never

14

Surge

Best case

Expected

Worst case

Never

Never

Vast increase

Best case

Expected

Worst case
Never

19

Never

25

Never

Never

Never

Never

27

No. of deaths

While waiting for

needed resources
While receiving needed resources

Note: ICU = intensive care unit. 


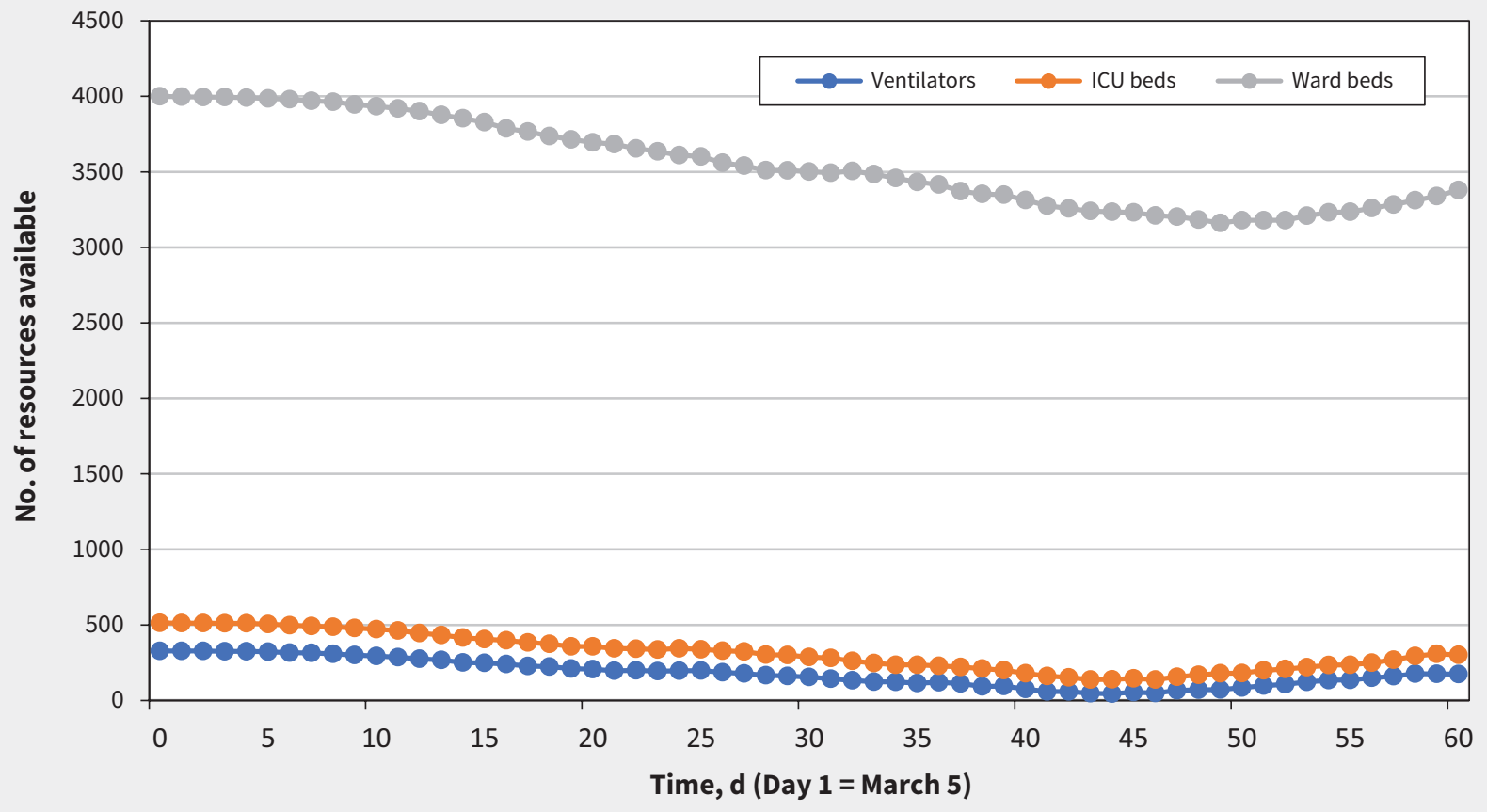

Figure 3: Resource depletion for the scenario with expected epidemic trajectory and base-case resources. ICU = intensive care unit.

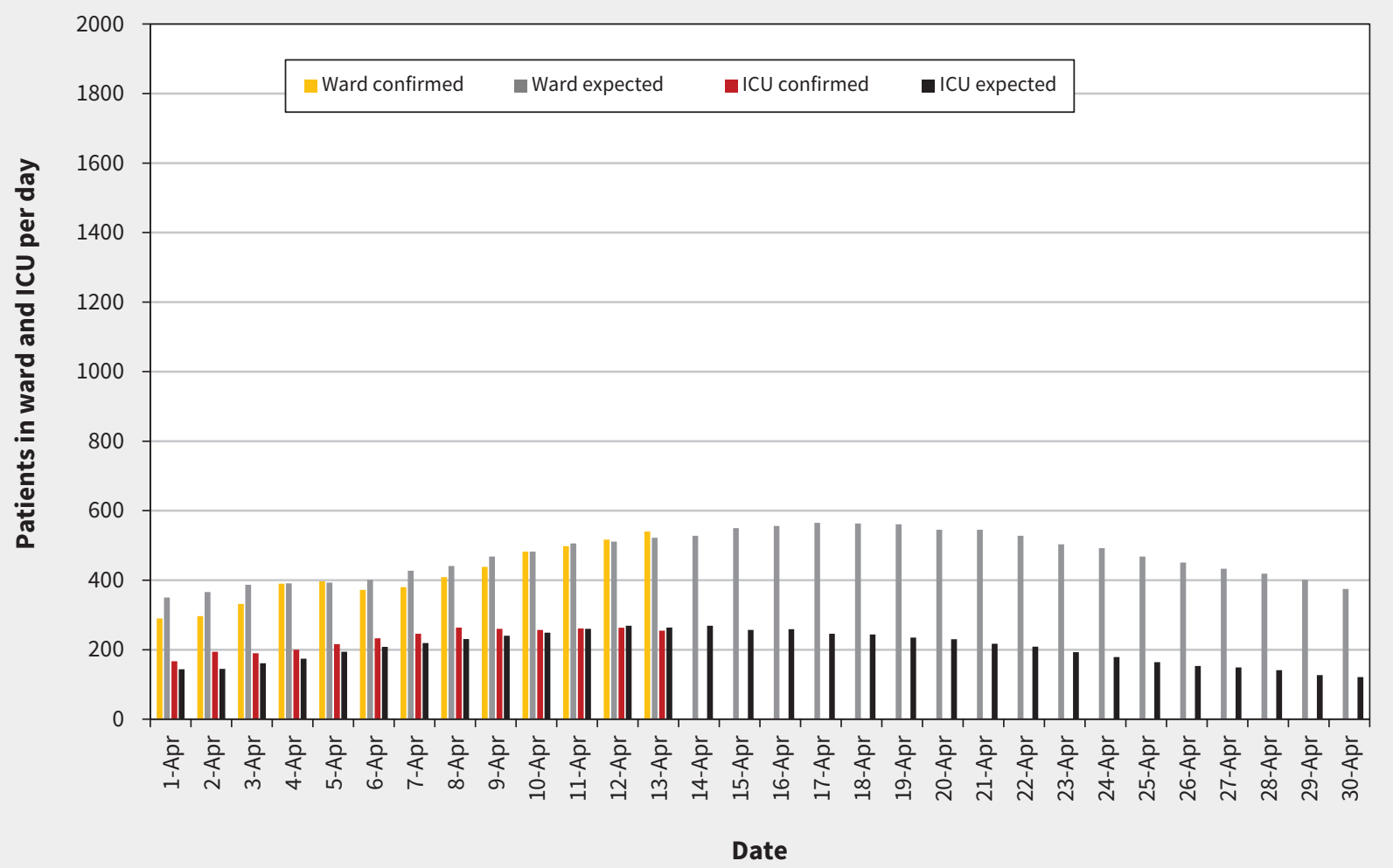

Figure 4: Expected and confirmed ward and intensive care unit (ICU) occupancy for patients with coronavirus disease 2019 (COVID-19) over time in Ontario. 
(Appendix 1). In this scenario, 353 patients died of COVID-19 despite having received appropriate care and hospital resources.

In the expected epidemic trajectory scenario, assuming basecase resources, our simulation predicted a total of 18047 COVID19 cases over 60 days, of which 3248 required admission to hospital. Using the observed data for Ontario, where physical distancing measures were implemented on Mar. 15, ICU beds, ventilator resources and ward beds in Ontario would not be depleted (Figure 3). In this scenario, 528 patients died of COVID19 despite receiving appropriate care and hospital resources.

After calibration, the projected occupancies of ICU and ward beds using this scenario compared favourably to the observed data in Ontario (Figure 4).

In the worst-case epidemic trajectory scenario, assuming base-case resources, our analysis predicted a total of 192839 COVID-19 cases over 60 days, of which 34711 required admission to hospital. Ontario's ICU bed and ventilator resources would have been depleted in about 19 and 14 days, respectively, from the start. Wards would have been unable to accommodate new patients in about 26 days (Appendix 1 ). In this scenario, with more daily cases of patients with COVID-19 infection and an earlier date of resource depletion, we predicted that 13321 patients would have died while waiting for appropriate care and 1060 patients who had received appropriate care would also have died.

\section{Resource expansion scenarios}

The worst-case epidemic trajectory scenario was the only scenario in which base-case resources were insufficient to meet demand. In this scenario, an increase in ICU beds and ventilator resources delayed but did not eliminate ICU bed and ventilator depletion (Appendix 1). For example, in the surge resource scenario, ICU beds and ventilators were depleted at about 25 and 19 days, respectively, instead of 17 and 14 days from the start date.

\section{Mortality}

Assuming base-case resources, there would be 13321 deaths among patients waiting for resources in the worst-case epidemic trajectory scenario. However, as the number of resources increased, the number of deaths among patients waiting for resources decreased to 8605 in the surge resource scenario and 1876 in the vast increase resource scenario (Table 2).

\section{Interpretation}

Our findings show that public health measures in conjunction with increased health system capacity substantially reduces or delays health system collapse and resource depletion.

As of Apr. 13, 2020, predictions of ICU occupancy from our model's expected epidemic trajectory scenario aligned well with observed data in Ontario, showing a stable ICU occupancy rate for patients with confirmed COVID-19 over 3 weeks (Apr. 6-27) and suggesting that public health interventions were effective. In contrast, had Ontario not taken these steps and followed Italy's trajectory, even with massive expansion of resource capacity, critical care capacity would have been overwhelmed quickly and catastrophically, and 13321 patients would have died because of lack of resources. Although Ontario has avoided a collapse of its hospital system, our model showed that even less strain on hospital resources and fewer deaths could have been possible in a best-case scenario resembling that of South Korea.

Two other models have studied COVID-19 in Ontario and Canada. ${ }^{10,16}$ Both developed transmission models to predict possible epidemic trajectories and forecast ICU occupancy but dealt with the resource availability at a relatively aggregated level. ${ }^{10,16}$ Our model explicitly considers resource constraints and accounts for surge in ICU capacity, enabling us to show the importance of excess deaths associated with resource depletion.

Our study has several strengths. In addition to modelling different pandemic scenarios, we also included a range of potential scenarios for an increase in hospital capacity, explicitly quantifying and forecasting these effects. Our estimates are different from others in that our estimates stratify mortality between patients who receive and do not receive adequate health care. Our study models patient flow through the hospital to estimate resource use and availability at each level of care, which allowed us to predict the effect of resource depletion on mortality. The validity of our study is strengthened by incorporating observed data from countries with early and aggressive public health measures and those in which these efforts were lacking. Finally, we obtained current estimates of local health care resources and used data from governmental and health care system proposals for increasing these resources in our estimates of potential capacity.

\section{Limitations}

Our study has several limitations. Our model relies on forecasting COVID-19 cases based on reported data, and on projections and assumptions about the effectiveness of physical distancing. Our assumption that patients requiring ward beds would not die from COVID-19, based on the current literature, may have led to underestimation of the number of in-hospital deaths. We assumed that the probability of deteriorating in the ward was zero to reflect the data available at the time of modelling and made the simplifying assumption that patients who were critically ill would be identified early within the emergency department and admitted directly to the ICU. We assumed emergency department resources could be expanded and did not consider emergency department capacity as a constraint. Actual data about the length of stay of patients with COVID-19 in the ward and ICU were not available at the time of modelling, and knowledge about the effect of insufficient resources on mortality was limited for patients with COVID-19.

Our model does not incorporate COVID-19 transmission in the hospital, potentially underestimating resource need. In addition, we did not explicitly consider constraints to health human resources or the availability of adequate personal protective equipment, and we assumed that all hospital resources were appropriately staffed and had necessary supplies.

Our study considered epidemic growth and resources for the whole of Ontario and did not consider regional differences within the province.

Finally, we acknowledge that deaths of patients in long-term care facilities in Ontario are concerning. Our model focused on 
acute care. Therefore, patients in long-term care who were transferred to hospital would have been captured in our model but not those who died in a long-term care facility.

\section{Conclusion}

Hospital resources in Ontario appear to be adequate to manage patients with COVID-19 because of the public health measures that were put in place, in conjunction with an increase in health system capacity. The counterfactual scenario in which these steps had not been taken is sobering, pointing to the need to maintain rigorous public health interventions in the near term. Premature relaxation of public health interventions will risk a resurgence of COVID-19 with the potential to overwhelm hospital resources in Ontario.

\section{References}

1. Naming the coronavirus disease (COVID-19) and the virus that causes it. Geneva: World Health Organization. Available: www.who.int/emergencies/diseases /novel-coronavirus-2019/technical-guidance/naming-the-coronavirus-disease -(covid-2019)-and-the-virus-that-causes-it (accessed 2020 Mar. 20).

2. WHO Director-General's opening remarks at the media briefing on COVID-19March 11 2020. Geneva: World Health Organization; 2020. Available: www.who. int/dg/speeches/detail/who-director-general-s-opening-remarks-at-the-media -briefing-on-covid-19---11-march-2020 (accessed 2020 Mar. 20).

3. COVID-19 situation update worldwide, as of 13 April 2020. Solna (Sweden): European Centre for Disease Prevention and Control; 2020. Available: www.ecdc.europa.eu/ en/geographical-distribution-2019-ncov-cases? (accessed 2020 Apr. 13).

4. Coronavirus disease (COVID-2019) situation reports. Geneva: World Health Organization; 2020. Available: www.who.int/emergencies/diseases/novel -coronavirus-2019/situation-reports (accessed 2020 Apr. 12).
5. Coronavirus disease 2019 (COVID-19): Situation report - 59. Geneva: World Health Organization; 2020. Available: www.who.int/docs/default-source/ coronavirus/situation-reports/20200319-sitrep-59-covid-19.pdf?sfvrsn=c3dcdef9 _2 (accessed 2020 Mar. 20).

6. COVID-19 Community Mobility Reports. Google. Available: www.google.com/ covid19/mobility/ (accessed 2020 Apr. 11).

7. Murthy S, Gomersall CD, Fowler RA. Care for critically ill patients with COVID-19. JAMA 2020 Mar. 11 [Epub ahead of print]. doi: 10.1001/jama.2020.3633.

8. Remuzzi A, Remuzzi G. COVID-19 and Italy: What next? Lancet 2020;395:1225-8.

9. Ferguson NM, Laydon D, Nedjati-Gilani G, et al. Imperial College COVID-19 Response Team. Report 9: Impact of non-pharmaceutical interventions (NPIs) to reduce COVID-19 mortality and healthcare demand. London (UK): Imperial College London; 2020.

10. Tuite AR, Fisman DN, Greer AL. Mathematical modelling of COVID-19 transmission and mitigation strategies in the population of Ontario, Canada. CMAJ 2020;192:E497-505.

11. Wu Z, McGoogan JM. Characteristics of and important lessons from the coronavirus disease 2019 (COVID-19) outbreak in China: summary of a report of 72314 cases from the Chinese Center for Disease Control and Prevention. JAMA 2020 Feb. 24 [Epub ahead of print]. doi: 10.1001/jama.2020.2648.

12. Hospital beds. Organisation for Economic Co-operation and Development; 2018. Available: https://data.oecd.org/healtheqt/hospital-beds.htm (accessed 2020 Mar. 25)

13. Coronavirus disease 2019 (COVID-19) daily epidemiology update. Public Health Agency of Canada; updated 2020 May 3.

14. COVID-19 updates. Critical Care Services Ontario; 2020. Available: https://critical careontario.com/covid-19-updates (accessed 2020 Apr. 14).

15. Bellani G, Laffey JG, Pham T, et al.; LUNG SAFE Investigators; ESICM Trials Group. Epidemiology, patterns of care, and mortality for patients with acute respiratory distress syndrome in intensive care units in 50 countries. JAMA 2016;315:788-800.

16. Shoukat A, Wells CR, Langley JM, et al. Projecting demand for critical care beds during COVID-19 outbreaks in Canada. CMAJ 2020;192:E489-96.
Competing interests: Kali Barrett has received personal fees from Xenios AG, outside of the submitted work. No other competing interests were declared.

This article has been peer reviewed.

Affiliations: Institute of Health Policy, Management and Evaluation (Barrett, Khan, Mac, Naimark, Sander), University of Toronto; University Health Network (Barrett, Khan, Ximenes); Toronto Health Economics and Technology Assessment (THETA) collaborative (Mac, Ximenes, Sander), University Health Network; Sunnybrook Hospital (Naimark), Toronto, Ont.

Contributors: Kali Barrett, Yasin Khan, Stephen Mac and Raphael Ximenes contributed equally and were joint primary authors. David Naimark and Beate Sander contributed equally and were joint senior authors. Stephen Mac, Raphael Ximenes and David Naimark constructed the model. Kali Barrett, Yasin Khan and Beate Sander acquired the data. All of the authors conceived the model, drafted the manuscript and revised it critically for important intellectual content, gave final approval of the version to be published and agreed to be accountable for all aspects of the work.

Funding: This research was supported, in part, by a Canada Research Chair in Economics of Infectious Diseases held by Beate Sander (CRC-950-232429).

Data sharing: The portion of study data derived from the literature and publicly available sources are available to other researchers. The portion derived from government registries are available by requests directed to those agencies

Accepted: May 4, 2020

Correspondence to: Beate Sander, beate.sander@theta.utoronto.ca 